\title{
Use of the flexible fibreoptic bronchoscope in diagnosis of sputum-negative pulmonary tuberculosis
}

\author{
PA WILLCOX, SR BENATAR, PD POTGIETER
}

From the Respiratory Clinic, Department of Medicine, University of Cape Town and Groote Schuur Hospital, Cape Town, South Africa

ABSTRACT In four years' use of the flexible fibreoptic bronchoscope in diagnosing sputumnegative pulmonary tuberculosis, of 275 patients with tuberculosis suspected from chest radiographic appearances, $89(32.4 \%)$ were shown to have active disease. In $60(67.4 \%)$ of these patients the diagnosis was made from samples obtained through the fibreoptic bronchoscope (56 from bronchial brushings, four from transbronchial biopsy samples). Of the 56 positive bronchial brushings, 35 were positive on direct smear and 21 only on culture. Transbronchial biopsy exclusively accounted for only four of the 60 positive diagnoses. In the remaining 29 patients, the diagnosis was made from further sputum or biopsy specimens in 15 , from the response to treatment in 12 , and at necropsy in two. In six of 10 patients with miliary tuberculosis, bronchial brushings were positive on direct smear.

The accurate diagnosis of active pulmonary tuberculosis is generally made by finding acid-fast bacilli in specimens of sputum. When a clinician is faced with a patient having a chest radiograph suggestive of active tuberculosis but with several sputum specimens negative for acid-fast bacilli on direct smear examination, two courses of action are open: either treatment may be started in the light of the patient's clinical condition, the result of the tuberculin test, and the radiographic appearances or the results of culture may be awaited and, if these are negative, the patient may be observed with regular radiographs and further sputum examination. More recently, with the advent of the flexible fibreoptic bronchoscope, a more aggressive approach to investigation has been adopted in such patients in an attempt to diagnose tuberculosis at an earlier stage in its natural history. As the pulmonary damage caused by tuberculosis can be minimised by early diagnosis and treatment, and as the amount of treatment required to achieve the desired effect probably depends on the extent of the disease at the time of diagnosis, such an approach has a sound clinical purpose. Surprisingly, little information is available from published reports on the value of this approach. In Cape Town pulmonary tuberculosis is still a common disease (250 new cases per 100000

Address for reprint requests: Professor SR Benatar, Department of University of Cape Town Medical School, Observatory 7925, South Africa. population notified a year). We have had the opportunity to evaluate the role of fibreoptic bronchoscopy in the diagnosis of sputum-negative suspected tuberculous lung disease over four years.

\section{Methods}

Two hundred and seventy-five patients who were suspected of having active pulmonary tuberculosis on the basis of abnormal chest radiographs but $\stackrel{x}{-}$ whose sputa were negative on repeated direct 3 smears, or who were unable to produce sputum, underwent bronchoscopy from August 1976 to Sep- $\frac{\rho}{3}$ tember 1980. Full details of the procedure have been described elsewhere. ${ }^{1}$ Bronchial brushings obtained from the relevant abnormal pulmonary $\frac{7}{2}$ segments were placed in normal saline, examined after Ziehl-Neelsen staining, cultured for mycobac- $\widetilde{N}$ teria, and inoculated into guinea pigs. Transbron- $N$ chial lung biopsy specimens were placed in $10 \%$ 오 formol saline and subsequently examined by $\omega$ haematoxylin and eosin and Ziehl-Neelsen stains. Whenever possible, postbronchoscopy sputum was collected for further examination.

\section{Results}

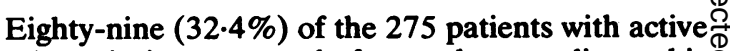
tuberculosis suspected from chest radiographic $\varrho$ appearances were subsequently shown to have active disease. Of these positive cases, $60(67.4 \%) \&$ 
Suspected active pulmonary tuberculosis

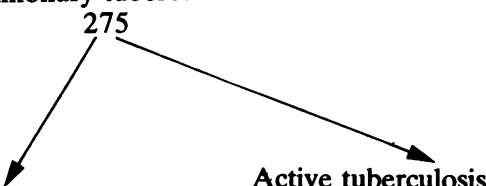

Active tuberculosis diagnosed $89(32.4 \%)$

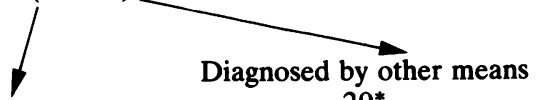

Diagnosis from

fibreoptic bronchoscopy

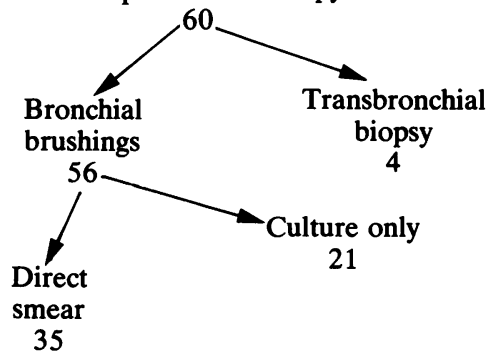

35

Outcome in 275 patients with active tuberculosis suspected from chest radiographic appearances.

*Sputum (7); response to antituberculous therapy (12), surgical biopsies (7), repeat bronchoscopy (1), necropsy (2).

were diagnosed from the specimens obtained by fibreoptic bronchoscopy and $29(32.6 \%)$ by other means (fig).

Adequate bronchial brushings were obtained in 83 of the 89 patients $(93 \%)$ and transbronchial biopsy specimens in $18(20 \%)$. Positive brushings were obtained in 56 patients, giving a diagnostic yield of $67.5 \%$. Transbronchial biopsy was positive in nine patients $(50 \%)$, five of whom also had positive brushings. Thus transbronchial biopsy was the exclusive means of making a diagnosis in only four patients (two with negative brushings and two from whom no brushings were obtained). Acid-fast bacilli were found on direct smear from brushings obtained from 35 patients, while in the remaining 21 patients acid-fast bacilli were only cultured from the brushings.

Of the 89 patients with proved tuberculosis, 10 (11\%) had miliary tuberculosis and in nine of these patients the diagnosis was made by fibreoptic bronchoscopy (table 1). Brushings were obtained in nine patients, yielding positive results in seven (78\%)six on direct smear and one on culture only. Three of the four transbronchial biopsy specimens were positive and in one patient both the biopsy and brushings were diagnostic.
Table 1 Diagnostic methods in patients with miliary tuberculosis

\begin{tabular}{ll}
\hline & No of patients \\
\hline Total patients & 10 \\
Miliary tuberculosis diagnosed & 9 \\
Failed diagnosis & 1 \\
Brushings performed & 9 \\
Brushings positive & 7 \\
Direct & 6 \\
Culture only & 1 \\
Brushings negative & 2 \\
Biopsy performed & 4 \\
Specimen positive & 3 \\
Specimen negative & 1 \\
Both brushings and biopsy specimen positive & 1 \\
\hline
\end{tabular}

Of the 29 patients in whom we failed to diagnose active pulmonary tuberculosis from bronchoscopic specimens, the diagnosis was established in 12 by appropriate response to antituberculosis therapy, from examination of sputum within 24 hours of bronchoscopy in three, from subsequent sputum specimens in four, at repeat bronchoscopy in one, from surgical biopsy specimens in seven (laparotomy one, thoracotomy four, lymph node biopsy two), and at necropsy in two patients.

Active pulmonary tuberculosis was excluded in 186 patients. Widely varying diagnoses were subsequently made in these patients (table 2 ). A confident diagnosis was made either by prolonged follow-up or by histological or cytological evidence of an alternative condition. Acute pneumonia was diagnosed in 40 patients $(22 \%)$, old inactive pulmonary tuberculosis in $33(18 \%)$, slowly resolving pneumonia in $23(12 \%)$, and malignant disease in $15(8 \%)$. Other pulmonary conditions diagnosed included aspergil-

Table 2 Diagnoses in patients in whom active pulmonary tuberculosis was excluded

\begin{tabular}{lc}
\hline & No $(\%)$ of patients \\
\hline Acute pneumonia & $40(22)$ \\
Old inactive pulmonary tuberculosis & $33(18)$ \\
Slowly resolving pneumonia & $23(12)$ \\
Malignant disease & $15(8)$ \\
Chronic inflammation & 12 \\
Bronchiectasis & 8 \\
Pyogenic lung abscess & 8 \\
Necrotising pneumonia & 5 \\
Fibrosis & 4 \\
Others & $38(20)$ \\
Total & 186 \\
\hline
\end{tabular}

lus infection (two cases), lymphoma (two), and septic pulmonary emboli (one case).

In four of the 89 patients with tuberculosis coexisting carcinoma was also diagnosed (4.5\%). 


\section{Discussion}

The use of the fibreoptic bronchoscope in an attempt to diagnose tuberculosis in patients with suspicious radiographic lesions but negative sputum specimens has not been widely reported. Only one other paper has described its value in any detail. ${ }^{2}$ The safety with which bronchial brushings and transbronchial biopsy specimens can be taken through the fibreoptic bronchoscope makes this an attractive method of investigating patients with radiographic lesions thought to be due to tuberculosis at a stage in the natural history before the tubercle bacilli are being expectorated. The advantage of making a diagnosis early in this way rather than waiting for the disease to progress until the sputum becomes positive lies not only in the prevention of pulmonary damage but also in the possibility of treating the disease at a time when a shorter course of chemotherapy may be adequate. Early diagnosis is even more important in the immunocompromised patient, and the safety of carefully performed fibreoptic bronchoscopy makes this an ideal investigation in such patients.

Active tuberculosis was ultimately diagnosed in one-third of the 275 patients in whom this diagnosis was considered on the basis of an abnormal chest radiograph. Although there are no other reports with which to compare our results, we believe that a diagnostic rate of $67 \%$ in the group of 89 patients ultimately shown to have tuberculosis is very satisfactory. In general, the radiographic lesions of suspected pulmonary tuberculosis were more amenable to brushing than to transbronchial biopsy-hence the greater number of brushings performed and the greater contribution of brushings to diagnosis.

Of the 56 patients in whom bronchial brushings contained acid-fast bacilli, these were identified on direct smear in $62.5 \%$ and on culture only in $37.5 \%$. Thus a positive diagnosis was frequently made rapidly and treatment could be started without delay. Our diagnostic yield from patients in whom tuberculosis was ultimately proved was significantly higher than that reported by Danek and Bower. ${ }^{2}$ In their study of 41 patients with tuberculosis whose sputum was negative on direct smear, they obtained positive results on direct smear of brushings in only eight patients $(20 \%)$. These authors made no mention of the frequency with which the diagnosis of tuberculosis may be missed even with bronchoscopy.

Transbronchial biopsies were performed in only 18 of our 89 patients and the specimens were positive in nine, giving a diagnostic yield of $50 \%$. Five of these patients also had positive results on bronchial brushings, while in the four remaining patients the diagnosis was made exclusively from the biopsy specimen. In the series reported by Danek and Bower transbronchial biopsy was carried out in 29 of the 41 patients, with positive results in eight $(28 \%) .^{2}$

The usefulness of collecting sputum in the hours immediately after bronchoscopy is indicated by the fact that in three of our patients the diagnosis was made from such specimens. Seven of 41 cases from the previously reported series were diagnosed from postbronchoscopy sputum, two from direct smear and five from culture. ${ }^{2}$ As lignocaine used for local analgesia before bronchoscopy has an inhibiting effect on the growth of mycobacteria, we support the recommendation that this agent should be used in the lowest possible quantities compatible with adequate analgesia. ${ }^{2}$

Miliary tuberculosis is associated with a high mortality rate in our community, death often occurring within 10 days of admission to hospital. ${ }^{3}$ As it is imperative that the diagnosis is confirmed as quickly as possible, we were encouraged to find that fibreoptic bronchoscopy provided a diagnosis on direct smear of bronchial brushings in six out of seven patients in whom brushings were positive on either direct smear or culture and in six out of nine patients in whom brushings were performed for suspected miliary tuberculosis. Transbronchial biopsy added to the diagnostic accuracy in such cases, two patients having the disease diagnosed exclusively on the basis of biopsy and one other having both a positive biopsy specimen and positive brushings.

In the 186 patients in whom tuberculosis was excluded various diagnoses were subsequently made, either on clinical grounds after careful follow-up or by specific histological or cytological study. The list of diagnoses was long, including uncommon conditions such as septic pulmonary emboli, aspergillus infection, and pulmonary alveolar proteinosis, to mention but a few. Acute pneumonia, old inactive tuberculosis, slowly resolving pneumonia, chronic inflammation, and malignant disease, however, accounted for $66 \%$ of diagnoses in these patients. In a community where $N$ tuberculosis is common and can mimic many other conditions radiographically, bacteriological proof is important if unnecessary antituberculous treatment is to be avoided.

The finding of coexistent tuberculosis and carcinoma in four of our patients is similar to previously described experience. Ting and colleagues found concomitant carcinoma in $5 \%$ of patients with tuberculosis. ${ }^{4}$ Other authors have noticed that carcinoma occurs 20 times more frequently in patients with tuberculosis than in the general population. ${ }^{5}$ The frequency with which this combination of diseases occurs should encourage a high degree of sus- 
picion and an active search for carcinoma in those patients with tuberculosis in whom any unusual features are present.

The value of any invasive procedure must be measured against its complication rate. In our previous report outlining the results of fibreoptic bronchoscopy in 705 patients, we reported no deaths and a very low morbidity rate associated with the procedure. There were no life-threatening haemoptyses and only two of seven pneumothoraces required tube drainage. ${ }^{1}$ This accords with previous reports. ${ }^{6}$

We conclude that flexible fibreoptic bronchoscopy is an extremely useful and a safe technique in evaluating patients in whom active tuberculosis is suspected from chest radiographic appearances but in whom examination of several sputum specimens has failed to identify acid-fast bacilli.

\section{References}

${ }^{1}$ Willcox PA, Benatar SR, Potgieter P, Ferguson AD, Bateman ED. Fibre-optic bronchoscopy-Groote Schuur Hospital experience. S Afr Med J 1981;60:651-4.

${ }^{2}$ Danek SJ, Bower JS. Diagnosis of pulmonary tuberculosis by flexible fibre-optic bronchoscopy. Am Rev Respir Dis 1979;119:677-9.
${ }^{3}$ Prout S, Benatar SR. Disseminated tuberculosis: a study of 62 cases. $S$ Afr Med J 1980;58:835-42.

${ }^{4}$ Ting YM, Church WR, Ravikrishnan KP. Lung carcinoma superimposed on pulmonary tuberculosis. Radiology 1976;119:307-12.

${ }^{5}$ Campbell RE, Hughes FA. The development of bronchogenic carcinoma in patients with pulmonary tuberculosis. J Thorac Cardiovasc Surg 1960;40:98-101.

${ }^{6}$ Credle WF Jr, Smiddy JF, Elliot RC. Complications of fibre-optic bronchoscopy. Am Rev Respir Dis 1974;109:67-72.

This work was supported by the University of Cape Town staff research fund. We would like to acknowledge the contribution of the departments of pathology and microbiology.

\section{Addendum}

After submission of this paper for publication, Wallace et al (Am J Med 1981;70:1189-94) reported their results with fibreoptic bronchoscopy in 56 patients with clinically suspected active tuberculosis and consistently negative sputum on direct smear examination. Of 22 patients (39\%) shown to have tuberculosis, $15(68 \%)$ had the diagnosis made from specimens obtained at bronchoscopy. 